\title{
Intoxicant use increased among Norwegian adolescents between 1992 and 2002, and attempted suicides increased among girls
}

Rossow I, Groholt B, Wichstrom L. Intoxicants and suicidal behavior among adolescents: changes in levels and associations from 1992 to 2002 . Addiction 2005; 100:79-88.

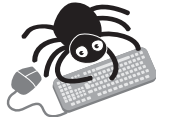

This article

contains extra text on the EBMH website

What is the prevalence of substance abuse and attempted suicides in Norwegian schoolchildren in 1992 and $2002 ?$

METHODS

$\square$

Design: Ecological study.

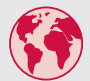

Setting: Norway; 67 schools in 1992 and 73 schools in 2002

으 Population: 11000 schoolchildren in 1992 and 12000 in 2002, aged 13-19 years. Exclusion criterion: a severe inability to read.

Assessment: Schoolchildren completed a questionnaire and were asked if they had ever attempted suicide or used intoxicants in the past year.

Fine

Outcomes: Prevalence of ever attempting suicide and intoxican use in the past year.

Follow up period: Ten years.

\section{MAIN RESULTS}

The prevalence of self-reported suicide attempts was significantly higher among girls in 2002 than 1992, with no significant difference among boys. Use of intoxicants in the past year was significantly higher among boys and girls in 2002 than 1992 (see http:// www.ebmentalhealth.com/supplemental for table).

\section{CONCLUSIONS}

Use of intoxicants increased among Norwegian schoolchildren from 1992 to 2002. Further studies are needed to establish if there is an association between intoxicant use and suicidal behaviour.

\section{NOTES}

No information was collected about the substance use patterns in schoolchildren who completed suicide. Multiple regression analysis found an independent association between intoxicant use and selfreported suicide attempts. After adjustment for independent risk factors, including depressive mood, loneliness, global self-worth, parental care, and living with both parents, intoxicant use was significantly associated with self-reported suicide attempts

For correspondence: Ingeborg Rossow, Norwegian Institute for Alcohol and Drug Research, POB 565 Sentum, N-0105, Oslo, Norway; email@ ir:strus.no

Sources of funding: The Norwegian Research Council and the Norwegian Institute for Alcohol and Drug Research. $(\mathrm{p}<0.001)$. However, the temporal relation between suicidal behaviour and intoxicant use was not examined, therefore causality cannot be assumed.

\section{Commentory}

Net he rate of adolescent suicidal attempts ranges from $2.8 \%$ in the Netherlands ${ }^{1}$ to $13.2 \%$ in British adolescents. ${ }^{2}$ The study by Rossow

et al investigated the trends in suicidal behaviour and substance abuse among 13-19 year old school children in the Netherlands. Suicidal attempts amongst students increased from $8.3 \%$ in 1993 (girls $=10.6 \%$; boys $=6.0 \%$ ) to $10.0 \%$ (girls $=13.6 \%$; boys $=6.2 \%$ ) in 2002. This is a statistically significant increase over a 10 year period, and highlights a higher prevalence of suicidal attempts among girls. The present report confirms previous epidemiological findings of an increased prevalence of suicidal behaviour: a similar trend in adolescent suicidal behaviour has been reported in America and Canada ${ }^{3}$ and also in developing countries such as Trinidad and Tobago. ${ }^{5}$

Rossow et al also identify a significant increase in substance abuse between 1992 and 2002. Although a causal relation cannot be assumed from Rossow et al's study because a temporal sequence between individuals' substance abuse and suicidal attempts was not established, it is interesting to note that a previous study reported the absence of alcohol abuse to be a protective factor in adolescent suicidal behaviour. ${ }^{5}$

This study should alert practitioners and researchers in all communities to the potential role of substance abuse in suicidal behaviour among adolescents. Although there may be variations in protective and risk factors in different cultures, ${ }^{6}$ the potential of the association between substance abuse and suicidal behaviour must be further investigated. Should a causal relation be found to exist, the early treatment of substance abuse would have an important role to play in the planning of suicidal behaviour interventions and related public health policy. Hari D Maharajh, FRCPsych, LLB, CMT Psychiatry Unit, Department of Clinical Medicine, University of the West Indies, Trinidad, West Indies

1 Tomori M, Keinhorst CWM, de Wilde EJ, et al. Suicidal behaviour and family factors among Dutch and Slovenian high school students: a comparison. Acta Psychiatr Scand 2001; 104:198-203

2 Hawton K, Rodham K, Evans E, et al. Deliberate self harm in adolescents: self report survey in schools in England. BMJ 2002;235:1207-1 1 .

3 Brener ND, Kruh EG, Simon TR. Trends in suicidal ideation and suicidal behaviours among high school students in the United States 1991-1997. Suicide Life Threat Behav 2000;30:304-12.

4 Edwards MJ, Holden RR. Coping, meaning in life and suicidal manifestations: examining gender differences. J Clin Psychol 2001;57:1517-34

5 Ali A, Maharajh HD. Social predictors of suicidal behaviour in adolescents in Trinidad and Tobago. Soc Psychiatry Psychiatr Epidemiol 2005;40: 186-91

6 Crompton MT, Thompson NJ, Kaslow NJ. Social environment factors associated with suicide attempt among low-income African Americans: The protective role of family relationships and social support. Soc Psychiatry Psychiatr Epidemiol 2005;40:175-85. 